Single trial analysis of slow cortical potentials: a study on anticipation related potentials

This article has been downloaded from IOPscience. Please scroll down to see the full text article.

2013 J. Neural Eng. 10036014

(http://iopscience.iop.org/1741-2552/10/3/036014)

View the table of contents for this issue, or go to the journal homepage for more

Download details:

IP Address: 80.238.234.181

The article was downloaded on 23/04/2013 at 23:23

Please note that terms and conditions apply. 


\title{
Single trial analysis of slow cortical potentials: a study on anticipation related potentials
}

\author{
Gangadhar Garipelli, Ricardo Chavarriaga and José del R Millán \\ Chair on Non-Invasive Brain-Machine Interface, Center for Neuroprosthetics, School of Engineering, \\ École Polytechnique Fédérale de Lausanne, Station 11, 1015 Lausanne, Switzerland \\ E-mail: Gangadhar.Garipelli@gmail.com, Ricardo.Chavarriaga@epfl.ch and Jose.Millan@epfl.ch
}

Received 3 November 2012

Accepted for publication 18 March 2013

Published 23 April 2013

Online at stacks.iop.org/JNE/10/036014

\begin{abstract}
Objective. Abundant literature suggests the use of slow cortical potentials (SCPs) in a wide spectrum of basic and applied neuroscience areas. Due to their low signal to noise ratio, these potentials are often studied using grand-average analysis, which conceals trial-to-trial information. Moreover, most of the single trial analysis methods in the literature are based on classical electroencephalogram (EEG) features $([1-30] \mathrm{Hz})$ and are likely to be unsuitable for SCPs that have different signal properties (such as having the signal's spectral content in the range $[0.2-0.7] \mathrm{Hz}$ ). In this paper we provide insights into the selection of appropriate parameters for spectral and spatial filtering. Approach. We study anticipation related SCPs recorded using a web-browser application protocol and a full-band EEG (FbEEG) setup from 11 subjects on two different days. Main results. We first highlight the role of a bandpass with [0.1-1.0] Hz in comparison with common practices (e.g., either with full dc, just a lowpass, or with a minimal highpass cut-off around $0.05 \mathrm{~Hz}$ ). Secondly, we suggest that a combination of spatial-smoothing filter and common average reference (CAR) is more suitable than the spatial filters often reported in the literature (e.g., re-referencing to an electrode, Laplacian or CAR alone). Thirdly, with the help of these preprocessing steps, we demonstrate the generalization capabilities of linear classifiers across several days (AUC of $0.88 \pm 0.05$ on average with a minimum of $0.81 \pm 0.03$ and a maximum of $0.97 \pm 0.01$ ). We also report the possibility of further improvements using a Bayesian fusion technique applied to electrode-specific classifiers. Significance. We believe the suggested spatial and spectral preprocessing methods are advantageous for grand-average and single trial analysis of SCPs obtained from EEG, MEG as well as for electrocorticogram. The use of these methods will impact basic neurophysiological studies as well as the use of SCPs in the design of neuroprosthetics.
\end{abstract}

S Online supplementary data available from stacks.iop.org/JNE/10/036014/mmedia

(Some figures may appear in colour only in the online journal)

\section{Introduction}

Slow cortical potentials (SCPs) are defined as the positive or negative deflections observed in electroencephalograms (EEGs) and magnetoencephalograms (MEGs), lasting from about a third of a second to several seconds with magnitudes up to $50 \mu \mathrm{V}[1,2]$. These potentials are frequently studied in various cognitive tasks (e.g., language [3]) as well as sensorymotor tasks (e.g., motor preparation [4] and expectation [5]). Abundant literature suggests their use in a wide spectrum of basic and applied fields in neuroscience, e.g., psychophysics, neuro-rehabilitation, clinical practice and brain-computer interfaces (BCIs) [6-8]. 
SCP modulations have been reported to encode movement specific parameters such as distance [9, 10], type and speed of real and imagined wrist movements $[11,12]$ and discrimination of torque rate [13]. Hence, they may also be used for decoding hand movement trajectories [14, 15]. Interestingly, SCPs have also been used to assess the role of the premotor cortex in distinguishing self-initiated and cue-initiated movement preparation [16]. Furthermore, in stroke patients, impairment specific signatures are observed in movement related SCPs, which could serve as diagnostic/prognostic measures for clinical practice [17].

Due to the inherent noise associated with the measurement of event related potentials (ERPs) in general and SCPs in particular (see section 2), most of the previous studies have been conducted by using averaging of a large number of trials. However, over the past three decades a few studies have reported successful real-time recognition of SCPs enabling subjects' self-regulation of these potentials via neuro-feedback $[18,19,2]$. Such methods have been shown to be useful for the design of BCI based tools for communication and control in severely paralyzed patients $[19,20,2]$, treating cognitive disorders (e.g., attention deficit hyperactivity disorder) and monitoring psychiatric conditions such as schizophrenia and depression [21]. These works, however, required relatively long time processing windows (around 8-10 s) to reliably estimate the SCPs.

A particular instance of SCP is associated with anticipatory behavior and can be observed in the classic contingent variation (CNV) paradigm [5]. We have previously explored its single trial recognition for BCI, designing methods for fast and online classification [22-24]. However, classification performance was largely varied across runs and subjects, sometimes staying at levels close to chance. In these studies, although the grand-average ERPs are clearly different for each condition, the trial distributions showed a large overlap.

In this paper we describe methods to enhance the signal to noise ratio (SNR) of SCPs and allow robust single trial recognition, which makes it particularly interesting for BCI applications as well as for basic neuroscience research. In particular, we systematically assess the effect of spectral and spatial filtering on the trial-to-trial variability of anticipatory SCPs. Using an assistive technology (AT) based web-browsing scenario, we report appropriate pre-processing steps that substantially reduced the noise associated with SCPs. These methods yield high classification accuracy (area under curve (AUC) of $0.88 \pm 0.05$ ). Furthermore, the methods show excellent generalization capabilities over several recording days (tested up to 15 days).

In the following section 2 we discuss the noise associated with the measurement of SCPs in both the spectral and spatial domains. We then describe the experimental protocol used to study the recognition of anticipation related SCPs on 11 subjects during different sessions (see sections 3.1 and 3.2). Next, we compare spectral filters appropriate for the SCP features as well as commonly used spatial filters (such as Laplacian and common average reference (CAR)) and new spatial-smoothing filters (see sections 3.3 and 3.4). Section 4.1 reports the effect of spectral filtering, while section 4.2 focuses on spatial filtering. Then, we report the single trial recognition of our methods using a single electrode $(\mathrm{Cz})$ over different recording days (section 4.3). We also provide insight into the possibility of improving classification performance by the fusion of electrode-specific classifiers, using a Bayesaverage method. Finally, in section 5 we discuss the results and implications of our methods and present our conclusions in section 6.

\section{Slow cortical potentials}

To address the trial-to-trial variability resulting from inherent background neural activity and a variety of non-neural artifacts, several computational methods have been proposed and tested on ERPs [25, 26]. However, a survey on signal processing methods for various electrical potentials for BCI applications [27], points out that only a few studies focus on SCPs. It is worth noting that in a recent attempt to detect a movement before it occurs, authors acknowledged the extreme difficulties associated with single trial analysis of SCPs, and avoided using them [28]. Most of the single trial analysis methods reported in the literature are designed in the context of classical EEG, where the features lie in the frequency range [0.5-30] $\mathrm{Hz}$. We argue here that the preprocessing methods designed for obtaining those features are suboptimal for the single trial analysis of SCPs which appear in the frequency range [0.2-0.7] Hz [29-31] and are usually recorded with FbEEG systems [32-34] or systems with close to dc highpass. The inclusion of the potentials close to dc raises a challenge due to the $1 / f$ scale-free statistical behavior of EEG [28, 35-37, 26]. More specifically, the task-irrelevant infraslow oscillations (ISOs) $\leqslant 0.1 \mathrm{~Hz}$ contribute significantly to the trial-to-trial variability. These fluctuations exhibit amplitudes up to $100 \mu \mathrm{V}$ or more, while event-related SCP grandaverages are observed only up to $15 \mu \mathrm{V}$ (see figure 1(a)) $[18,26]$. Figure 1(b) illustrates the inherent variability of the FbEEG trials of the SCP recordings conducted for this study. Furthermore, contrary to the SCP grand-averages, which have relatively homogeneous scalp distributions (see figure 3), single trials exhibit high frequency spatial noise, which is likely to result from local changes in skin conductance (see figure 1(c)) [32, 18, 38]. In other words, nearby electrodes show different trends in single trials as compared to the event related SCP grand-averages. In summary, the spectral and spatial variability pose an obstacle for the single trial analysis of SCPs.

Although some of the above challenges were reported in previous early studies [32, 18, 38], the influence of these problems remains underestimated (a wide variety of spectral choices have been employed in the literature; see supplementary table 1, available from stacks.iop.org/JNE/10/036014/mmedia). In most reports, the high-pass filtering is either ignored or set to $0.05 \mathrm{~Hz}$ [39-44]. In some other studies dc removal is achieved by simple de-trend algorithms [32, 40, 10, 9]. Given the spectral localization of SCPs in the range [0.2-0.7] Hz, these filters are likely to be sub-optimal due to the inclusion of a broad spectral range. 


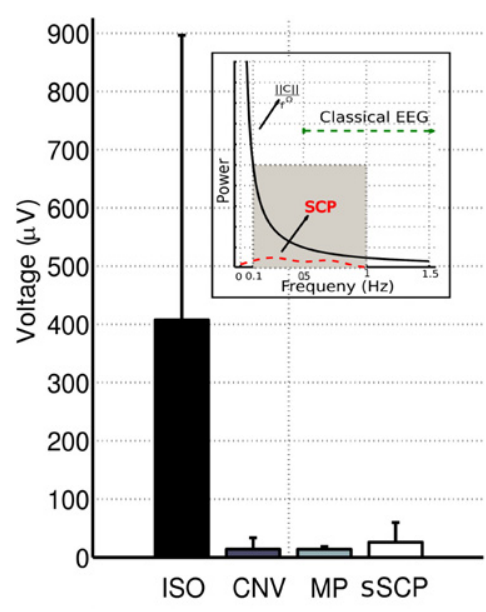

(a)

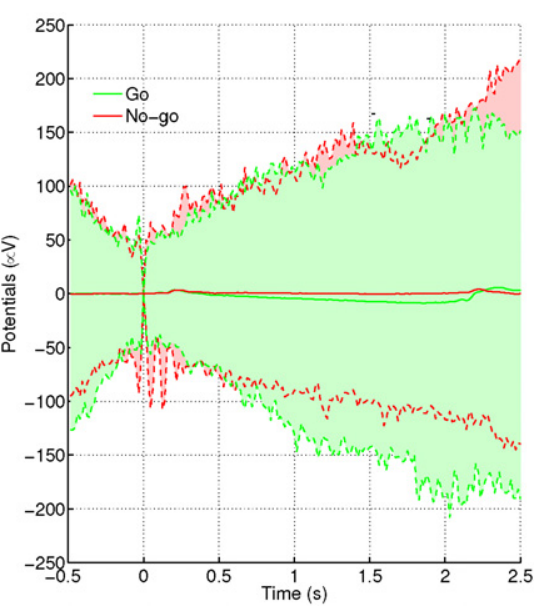

(b)
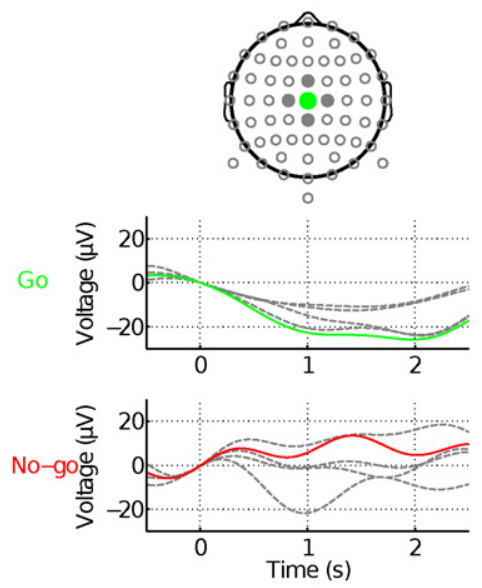

(c)

Figure 1. The SCPs' signal characteristics. (a) The magnitude of ISOs compared with different forms of event-related SCPs (in the plot, sSCP stands for self-regulated SCPs). The statistics are obtained from previously reported studies, [28, 2, 4, 19, 38, 50-52, 33, 34]. The inset is an illustration of the relative power of SCPs and the $1 / f$ statistic of FbEEG [35-37] ( $\Omega \backsim 1$ and $\mathbf{C}$ is a constant). The background ISOs below $0.1 \mathrm{~Hz}$ exhibit much larger amplitudes compared to SCP magnitudes [34]. (b) Variance of anticipatory SCPs (at Cz electrode) used in the current study. (c) Spatial variability observed in a typical single trial for the Cz electrode (Go trial in green, or No-go trial in red) and its four neighboring electrodes (in gray) spectral filtering is $0.1-1 \mathrm{~Hz}$ (see section 3 ).

More recently a few studies have reported a bandpass filter in the range $[0.11] \mathrm{Hz}[45,46,15]$. The appropriate choice of spectral range for the single trial analysis of SCPs has been elusive. Furthermore, a significant number of studies are conducted using a single electrode (usually a vertex electrode) $[20,19,2,47]$. In the studies using multiple recording sites, the spatial filtering is either skipped or performed using Laplacian filters or CAR [39, 22, 48, 49]. Given the homogenous nature of the SCP scalp distribution, both of these approaches are likely to be sub-optimal. In this paper, we systematically study the choice of appropriate spectral and spatial filters for the single trial analysis of SCPs.

\section{Methods}

In this section we describe the experimental setup used for recording anticipation related potentials, FbEEG acquisition, preprocessing parameters and classification methods.

\subsection{Experimental set-up}

The protocol corresponds to an AT software for web browsing by icon-selection with an auto-scanning mode, as shown in figure 2 (the scanning of icons was similar to that of [53]). It is a variant of the $\mathrm{CNV}$ paradigm, in which one or more contingent warning stimuli predicted an imperative stimulus resulting in a sequence. A cue presented $2 \mathrm{~s}$ prior to the beginning of each sequence indicated the target icon. In each sequence, the icons were highlighted sequentially every $2 \mathrm{~s}$ starting with the Twitter icon, resulting in 1-4 trials of $2 \mathrm{~s}$ length (a trial corresponds to the time window between the highlight of two consecutive icons). In each sequence, subjects were asked to press a button as quickly as possible using their right hand after the target icon was highlighted-thus requiring anticipation from the moment of the highlight of the pre-target icon. The time window from the pre-target icon highlight to the target icon highlight is considered to be a Go trial. Any other trial, where the subsequent icon is not the target, is a No-go trial. Reaction time (RT) and the web-page corresponding to the target icon were presented to the subjects if the RT $\leqslant \pm 100 \mathrm{~ms}$ (thus motivating the subjects to anticipate). If this criterion was not met, the scan finished approximately $1 \mathrm{~s}$ after with feedback showing an empty web-page. The scanning order and the positions of the icons remained constant throughout the experiment. To minimize artifacts, the subjects were instructed to fix their eyes on a ' + ' symbol presented in the middle of the computer screen and to avoid any other movements, such as of the facial muscles, tongue or head, during the scan period.

\subsection{FbEEG acquisition and ERP analysis}

We recorded FbEEGs of 11 healthy human subjects (age $26.4 \pm 2.4$ years, 1 female, all right-handed) with an average of $123 \pm 28$ Go trials and $264 \pm 68$ No-go trials per subject per session over a minimum of two sessions with a gap of 17 days. Two of the subjects also participated in a third session (13 and 7 days after first session). The data were acquired with 64 electrodes according to the international extended 10-20 standard along with three electrooculogram (EOG) electrodes and two electromyogram (EMG) electrodes as shown in figure 2(c) using a Biosemi Inc. ActiveTwo amplifier. The three EOG electrodes were used to derive horizontal, vertical and radial components $[54,55]$. The EMG was computed using the bipolar derivation of the two electrodes placed on the forearm extensor digitorum muscle. The electrode locations were chosen to capture the activity related to the right index finger. No special skin preparation, such as perforation or scraping was performed, except that the electrode offsets were kept below $25 \mathrm{mV}$ by applying SignaGel (Parker Laboratories Inc.), to achieve good contact with the skin. The signals were 


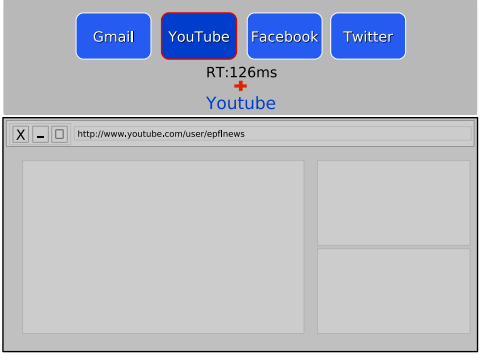

(a)

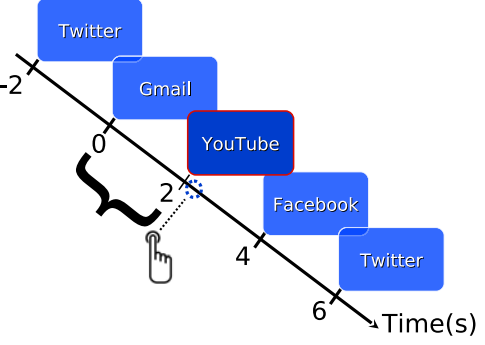

(b)

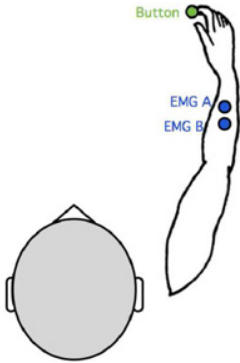

(c)

Figure 2. Experimental protocol: an AT framework for web-browsing. This protocol is a variation of the classical CNV paradigm with sequential warning stimuli. (a) A snapshot of the computer screen presented to the subjects. (b) Time-line of events. Icons are sequentially highlighted every $2 \mathrm{~s}$ (the target icon is YouTube in this example). The Go trial corresponds to the time window from the highlight of the Gmail icon at $0 \mathrm{~s}$ until the highlight of the YouTube icon at 2 s. (c) The placement of EEG, EOG and EMG electrodes.

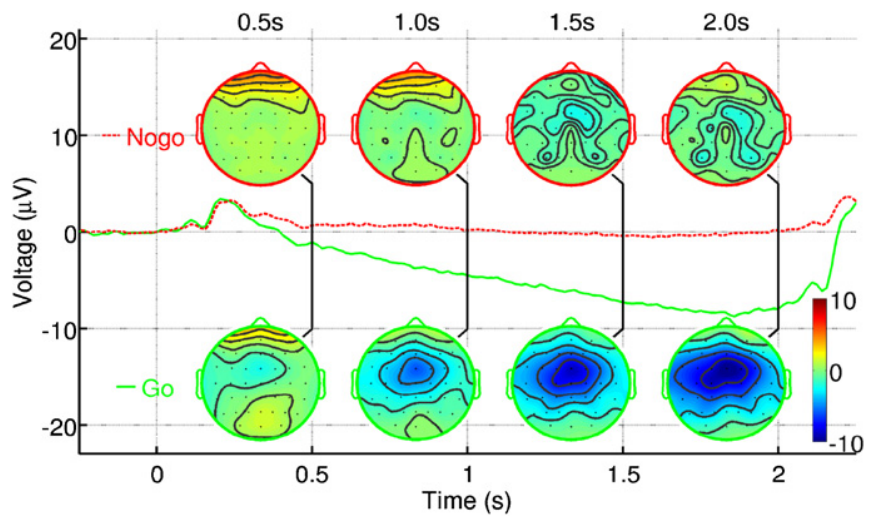

Figure 3. Scalp topographies of Go (bottom; green line) and No-go (top; red line) conditions and $\mathrm{Cz}$ electrode grand-average potentials at different time points.

acquired with a low-pass cut-off at $400 \mathrm{~Hz}$ at $2 \mathrm{KHz}$ sampling rate. The acquired signals were zero-phase low-pass filtered with a cut-off frequency of $25 \mathrm{~Hz}$ and then down-sampled to $64 \mathrm{~Hz}$. Depending on the spectral exploration described in section 3.3 , the data were further bandpass filtered. The trials were then extracted with $\left[\begin{array}{ll}-0.5 & 2.5\end{array}\right] \mathrm{s}$ windows, synchronized to the highlighting of icons and labeled accordingly (0 s corresponds the to highlight of pre-target icon (see figure 2(b)). The data were baseline corrected using the sample at $0 \mathrm{~s}$. The trials that had a maximum magnitude (at any electrode) above $250 \mu \mathrm{V}$, were assumed to contain electrode contact artifacts and thus discarded from the study ( $\approx 1 \%$ of total trials).

The data were re-referenced to $\mathrm{Oz}$ electrode potentials, where anticipation related SCPs were expected to have the lowest magnitude due to polarity shift [1]. Grand-averages computed across all subjects and days exhibited the wellknown CNV potential: an increasing negativity at the frontocentral electrodes (maximal at $\mathrm{Cz}$; approx. $10 \mu \mathrm{V}$ at $1.7 \mathrm{~s}$ ) for the Go condition and an almost flat response for the No-go condition (see figure 3). A slight asymmetry toward the left hemisphere can be observed, presumably due to hand motor preparation during the later part of the trial. The topographies reflect the low spatial frequency nature of the SCPs (i.e., the rate of change of potentials in adjacent electrodes is small), contrary to the single trials shown in figure 1(c).

\subsection{Spectral and spatial filtering}

The CNV Go No-Go potentials are clearly separable at the level of grand-averages (see figure 3), but the single trials suffers high variability (see figure 1(b)) due to high amplitude ISOs and high-frequency spatial noise (i.e., adjacent electrodes contain different SCP trends; see figure 1(c)). To assess the effect of both spectral and spatial filtering we define a separability index $f$ (see section 3.4) that represents the discriminability of Go and No-go potentials estimated at the $\mathrm{Cz}$ electrode.

We systematically explored parameters for spectral filtering (using zero phase finite impulse response (FIR) filters with order $N=10 x$ sampling rate to ensure a sharp transition; designed using fir 1 and applied using filtfilt routines of Matlab software, Mathworks Inc., USA). We investigated four categories of filters. In the first category we studied three filters-NO filtering (raw potentials), a low-pass filter with a cut-off at $5 \mathrm{~Hz}$ and a bandpass of [0.01-5] Hz. This category is similar to the commonly applied filtering parameters, where no filtering or just lowpass filtering is applied (supplementary table 1, available from stacks.iop.org/JNE/10/036014/mmedia). In the second category we fixed the low-pass cut-off at $1 \mathrm{~Hz}$ while setting the high-pass cut-off to $0.01 \mathrm{~Hz}, 0.05 \mathrm{~Hz}, 0.1 \mathrm{~Hz}, 0.2 \mathrm{~Hz}$, $0.3 \mathrm{~Hz}, 0.4 \mathrm{~Hz}$, and $0.5 \mathrm{~Hz}$. For the third category we fixed the low-pass cut-off at $2 \mathrm{~Hz}$ and varied the low-pass from 0.1 to $0.5 \mathrm{~Hz}$ in steps of $0.1 \mathrm{~Hz}$. The fourth category is similar to the third category, where the high-pass cut-off was fixed at $3 \mathrm{~Hz}$. These spectral categories were chosen to identify the appropriate candidates for high-pass cut-off and low-pass cut-off frequencies suited to the discriminability of CNV.

We also explored various spatial filters for the spectral filters described above. Again, the potentials at the $\mathrm{Cz}$ electrode were used for this study. First, we compared small Laplacian filter (SLAP), a large Laplacian filter (LLAP), CAR and no spatial filtering (just referencing to $\mathrm{Oz}$ electrode, Oz-REF). Given a recording at the $i$ th electrode, $e_{i}(t)$, CAR returns $e_{i}^{\mathrm{CAR}}(t)=e_{i}(t)-\frac{1}{N} \sum_{j}^{N} e_{j}(t)$, where $N$ is 
the number of electrodes $(=64)$. The Laplacian filtered data were computed as $e_{i}^{\text {Laplace }}(t)=e_{i}(t)-\frac{1}{K} \sum_{j}^{K} e_{j}(t)$. Where $j$ is the index of neighboring electrodes $(K=4)$ chosen in 'plus (+)' configuration around electrode $i$ [56]. For the SLAP the immediate neighboring electrodes were chosen, whereas for LLAP the second neighboring electrodes were chosen. Second, we tested a weighted average filter (WAVG), which is an anti-Laplacian filter, where the average neighboring activity is added rather than subtracted (i.e., $e_{i}^{\mathrm{WAVG}}(t)=e_{i}(t)+\frac{1}{K} \sum_{j}^{K} e_{j}(t)$, where the chosen electrodes are similar to the SLAP configuration). This filter was applied to CAR referenced data. Third, we studied a generalized version of the WAVG filter, the spatial smoothing filter (SSF), which was obtained by the spatial convolution of SCP data with a Gaussian kernel with the CAR referenced data. The $e_{i}^{\mathrm{SSF}}(t)=\sum_{j}^{K} w_{i j} e_{j}(t)$, where $w_{i j}=\exp \left(-\frac{d_{i j}^{2}}{2 \sigma^{2}}\right)$ and $d_{i j}$ was the Euclidean distance between electrodes $i$ and $j$ in $3 \mathrm{D}$ coordinates of a unit sphere fitted to the average head model (we assume spatially symmetrical distribution of SCP surrounding the selected electrode). The meta-parameter $\sigma$ value was obtained from training data as described in section 4 . The rationale of this smoothing filter is that, given a measurement of SCP source $s(t)$ at electrode $i, e_{i}(t)=$ $w_{i} s(t)+n(t)$, where $n(t) \sim \mathcal{N}(0, \sigma)$ and $w_{i}$ is a positive fraction that represents the extent to which $i$ th electrode may carry the signal components $s(t)$. The spatial smoothing filters are treated as a way to reconstruct $s(t)$, where $s^{\prime}(t)=w_{i} e_{i}(t)$, with $\left\langle s^{\prime}(t)\right\rangle=w_{i}\langle s(t)\rangle+w_{i}\langle n(t)\rangle$, which is equivalent to a scaled $\langle s(t)\rangle$ (ie. $\left\langle s^{\prime}(t)\right\rangle=w_{i}\langle s(t)\rangle$ ). The key difference between the smoothing filter and the Laplacian filter is that the later removes a fraction of the common activity of adjacent electrodes, thus increasing the spatial resolution, whereas the former reduced the unshared activity. In other words, SSF filters act as spatial low-pass filters or blurring kernels. Such Gaussian kernel based smoothing filters are widely used in the analysis of fMRI data [57], but to the best of our knowledge, these filters have not been explored in the analysis of SCPs.

\subsection{Feature selection and classification}

We selected features from the $\mathrm{Cz}$ electrode under which the $\mathrm{CNV}$ potentials are more prominent. For each trial, the potentials at eight equally spaced time points (i.e., at $0.25 \mathrm{~s}$, $0.5 \mathrm{~s}, \ldots, 2.0 \mathrm{~s}$ ) were concatenated and chosen as a feature vector, $\mathbf{x}=\left[e_{C z}\left(T_{1}\right) e_{C z}\left(T_{2}\right) \ldots e_{C z}\left(T_{8}\right)\right] \in R^{8}$ where, $T_{k}$ represents $k$ th time point. Based on preliminary analysis the number of features was restricted to eight. The small number of features sufficiently represents the evolution of an SCP in a $2 \mathrm{~s}$ window, while allowing one to properly estimate the feature distributions even in the case of a reduced number of samples. These feature vectors were used to compute a separability index $f$ for Go and No-go features as well as in training and testing the linear classifiers. To compute this index, the feature vectors were projected into a canonical space $y \in R$ by using $y=\mathbf{w}^{T} \mathbf{x}$ for better separation. The projection matrix $\mathbf{w}$, maximizes the between-class variance whilst simultaneously minimized the within-class variance [58]. Using the projected data, the separability index is computed as

$$
f=\frac{\left(\mu^{\mathrm{Go}}-\mu^{\mathrm{Nogo}}\right)^{2}}{\left(\sigma^{\mathrm{Go}}\right)^{2}+\left(\sigma^{\mathrm{Nogo}}\right)^{2}}
$$

where, $\mu$ and $\sigma$ are the mean and standard deviation computed in the canonical space for both Go and No-go trials. This score can be interpreted as 'the extent to which a linear classifier can discriminate the two distributions'. In other words, this score is used as a measure to predict the performance of the linear classifiers described below.

For the single trial classification of these SCP features we made the following assumptions. (1) The feature vectors of the Go and No-go classes follow unimodal Gaussian distributions. (2) Both Go and No-go classes have the same covariance matrices that are estimated from training data (we did not made an assumption of feature independence). (3) The training samples represented, to a fair extent, the underlying distribution of the Go and No-go conditions and did not change over time (from day to day). Based on these assumptions, we used a linear discriminant analysis (LDA) classifier for the single trial classification of a feature vector $\mathbf{x}$. Assuming a prior probability $p\left(\mathcal{C}_{\mathrm{Go}}\right)(=0.5$ in the absence of any knowledge), the posterior probability $p\left(\mathcal{C}_{\mathrm{Go}} \mid \mathbf{x}\right)$ is computed using the Bayes rule [58],

$$
\begin{gathered}
p\left(\mathcal{C}_{\mathrm{Go}} \mid \mathbf{x}\right)=\frac{p\left(\mathbf{x} \mid \mathcal{C}_{\mathrm{Go}}\right) p\left(\mathcal{C}_{\mathrm{Go}}\right)}{\sum_{k \in\{\mathrm{Go}, \mathrm{NoGo}\}} p\left(\mathbf{x} \mid \mathcal{C}_{k}\right) p\left(\mathcal{C}_{k}\right)} \\
p\left(\mathbf{x} \mid \mathcal{C}_{k}\right)=\frac{1}{(2 \pi)^{\frac{d}{2}}|\boldsymbol{\Sigma}|^{\frac{1}{2}}} e^{-\frac{1}{2}\left(\mathbf{x}-\mu_{k}\right)^{T} \Sigma^{-1}\left(\mathbf{x}-\mu_{k}\right)}
\end{gathered}
$$

where $\mu_{k}$ were mean vectors of Go and No-go classes. The shared covariance matrix was computed as $\boldsymbol{\Sigma}=$ $\frac{1}{2}\left(\boldsymbol{\Sigma}_{\mathrm{Go}}+\boldsymbol{\Sigma}_{\mathrm{No}-\mathrm{go}}\right)$. $d$ was the number of dimensions of the feature vector $\mathbf{x}(=8)$. The feature vector $\mathbf{x}$ was assigned to the Go class if the posterior $p\left(\mathcal{C}_{\mathrm{Go}} \mid \mathbf{x}\right) \geqslant \theta$.

Single trial classification performances are often evaluated using measures such as accuracy or error rates. However, these measures depend on the prior probabilities and the threshold $\theta$, which could only be inferred from a particular application-based loss function. To avoid the dependency on $\theta$, we computed the AUC of the receiver operating characteristics (ROC) graph. This measure represents 'the probability that the classifier will rank a randomly chosen positive instance higher than a randomly chosen negative instance' [59, 60]. We further obtained the confidence on the estimated AUC using a $k(=10)$ fold cross-validation technique [59]. The confidence on the averaged ROC graph was obtained by computing the standard deviation over folds of the true positive rate (TPR) and the false positive rate (FPR) at each threshold (i.e., $0 \leqslant \theta \leqslant 1$ ). The maximum bound on the standard error (SE) in estimating the AUC measure was computed taking into account the number of class-specific samples [60].

\section{Results}

\subsection{Spectral filtering}

Figure 4(a) shows the separability index, $f$, computed on data from day 1 for the four spectral filter categories of 


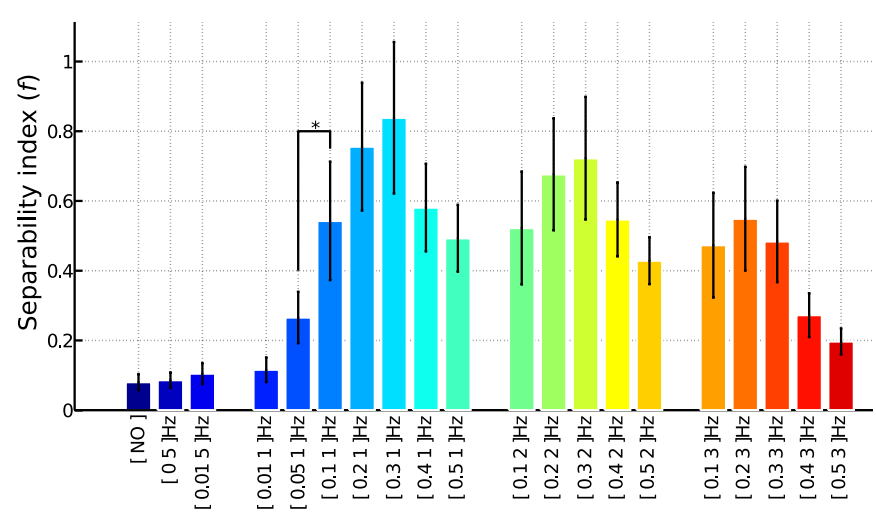

(a)

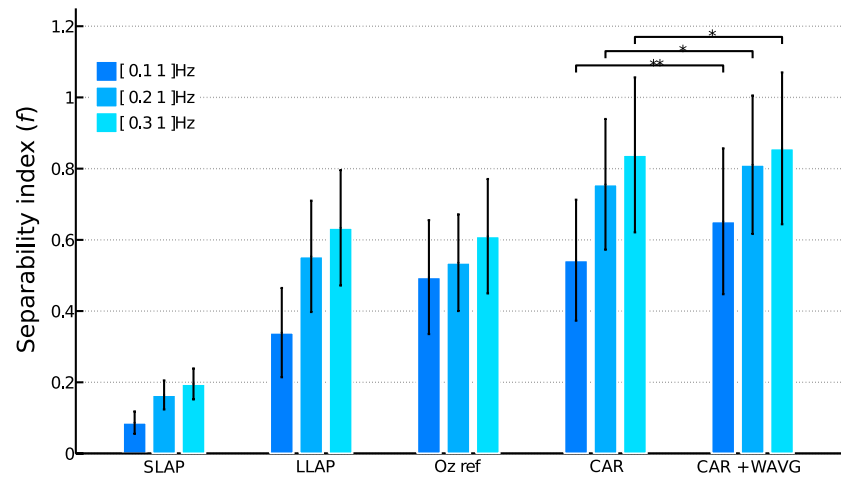

(b)

Figure 4. Separability index $f$ is compared for various spectral and spatial filter parameters using the data of day 1 . (a) The $f$ value is compared for CAR referenced signals filtered in various spectral pass-bands. The bars are clustered according to the categories described in section 3.3. (b) The $f$ value is compared for various spatial filters for the three best spectral filters. The thin vertical lines over the bars represent the SE computed over 11 subjects. Wilcoxon statistics are shown for selected cased with $p \leqslant 0.05^{*}$ and $p \leqslant 0.01^{* *}$.

each subject. For simplicity, the results of the spectral exploration are shown only for CAR filtered signals (similar patterns were obtained for other spatial filters, as shown in supplementary figure 1 , are available from stacks.iop.org/JNE/10/036014/mmedia). It is evident that the first category, wide-bandpass filters (raw signals, low-pass filter with cut-off at $5 \mathrm{~Hz}$ and bandpass of $0.01-5 \mathrm{~Hz}$; similar to common practices, see supplementary table 1, available from stacks.iop.org/JNE/10/036014/mmedia) resulted in a low separability index (with $f=0.08 \pm 0.02,0.09 \pm 0.02$, and $0.10 \pm 0.03$ ) as compared to the other categories. In the second category, where the low-pass cut-off is fixed at $1 \mathrm{~Hz}$ and the high-pass cut-off is varied, the $f$ value resulted in a gradual increase from $0.01 \mathrm{~Hz}$ till $0.1 \mathrm{~Hz}$, with the highest increase observed at $0.1 \mathrm{~Hz}(f=0.54 \pm 0.17)$ and the peak value at $0.3 \mathrm{~Hz}(f=0.83 \pm 0.21)$. As we further increased the high-pass cut-off toward $0.5 \mathrm{~Hz}$, the $f$ gradually reduced ( $f=0.49 \pm 0.10$ for $0.5-1 \mathrm{~Hz}$ bandpass filter). The $f$ is significantly higher for a high-pass filter with cut-off at $0.1 \mathrm{~Hz}$ than with $0.05 \mathrm{~Hz}(f=0.26 \pm 0.07)$ (Wilcoxon, $p \leqslant 0.05)$. The improvement for $0.3 \mathrm{~Hz}$ as compared to $0.1 \mathrm{~Hz}$ or $0.2 \mathrm{~Hz}$ ( $f=0.75 \pm 0.19)$ was not significant. Similar patterns of improvement until $0.3 \mathrm{~Hz}$ were observed also for the third and fourth categories, in which the low-pass cut-offs were fixed at 2 and $3 \mathrm{~Hz}$ respectively. However, overall $f$ values are higher for the second category compared to the rest of the categories. This observation is evident for filters with high-pass cut-off above $0.2 \mathrm{~Hz}$, whereas for filters with high-pass cut-off at $0.1 \mathrm{~Hz}$, similar $f$ values resulted.

In summary, $f$ is higher for the filters whose pass-band was a subset of $0.1-1 \mathrm{~Hz}$. Note the reduction of $f$ values as the high-pass increased from 0.3 to $0.5 \mathrm{~Hz}$. These observations reinforced that the task specific SCPs were localized in the frequencies above $0.1 \mathrm{~Hz}$ and the increasing high-pass results in less discriminant SCPs. Furthermore, a slight reduction in the high-pass below $0.1 \mathrm{~Hz}$ resulted in a significantly lower $f$, which is likely to be due to the noise injected by the high amplitude ISOs below $0.1 \mathrm{~Hz}$. Similarly, the inclusion of frequencies above $1 \mathrm{~Hz}$ is unlikely to provide more discriminable information for this task. These conclusions also hold for other spatial filter types (see figure 4(b)).

\subsection{Spatial filtering}

The separability index $f$ of spatial filters SLAP, LLAP, Ozreference, CAR and CAR+WAVG is shown in figure 4(b) for selected spectral filters (i.e., [0.1-1] Hz, [0.2-1] $\mathrm{Hz}$ and $[0.3-1] \mathrm{Hz})$. For all the compared spectral filters $([0.1-1] \mathrm{Hz}$, $[0.2-1] \mathrm{Hz}$ and $[0.3-1] \mathrm{Hz})$, the widely used SLAP $(f=0.08 \pm$ $0.03,0.16 \pm 0.04$ and $0.2 \pm 0.04$, respectively) performed worse compared to Oz-REF ( $f=0.5 \pm 0.16,0.50 \pm 0.11$ and $0.6 \pm 0.16$, respectively). This is likely due to the fact that the SCPs are homogeneously distributed activities across large scalp areas, and SLAP, by design, reduces this type of signal. Furthermore, the LLAP filter resulted in similar values of $f(=0.34 \pm 0.12,0.5 \pm 0.16$ and $0.6 \pm 0.16)$ to $\mathrm{Oz}-$ REF (no significant differences). In contrast, the CAR filtered signals resulted in a better $f(=0.54 \pm 0.17,0.75 \pm 0.18$ and $0.8 \pm 0.21$ ) as compared to Oz-REF. Thus CAR seems a better choice over the Laplacian filters to reduce global activity. However, as SCPs are broadly spread across the scalp, they are likely to influence the estimation of CAR. Therefore, this filtering may also reduce SCP activity. This shortcoming is partly addressed by applying the WAVG filter to the CAR filtered signals. This operation results in a better separability $f\left(=0.6 \pm 0.20^{* *}, 0.8 \pm 0.2^{*}\right.$ and $\left.0.86 \pm 0.2^{*}\right)$ than CAR alone (Wilcoxon, $p \leqslant 0.05^{*}$ and $p \leqslant 0.01^{* *}$ ). Note that the neighboring electrodes and the magnitude of the weights for WAVG filters are similar to a Laplacian filter. To obtain optimal weights for all the neighboring electrodes without any restriction, we applied SSF with different $\sigma$ values of the Gaussian kernel (see section 3.3) used in the convolution. The results of $f$ for various $\sigma$ are shown in figure 5 for the spectral filtering in the range from $0.1-1 \mathrm{~Hz}$. The highest values for $f$ were observed at $\sigma=0.15$ and resulted in slightly higher performance than CAR+WAVG of day $1(f=0.66 \pm 0.2)$. Similar results are also obtained for spectral filters with passband in the range from $0.2-1 \mathrm{~Hz}$ and $0.3-1 \mathrm{~Hz}$.

\subsection{Classification performance}

For the classification of Go/No-go potentials, we chose the spectral filter with pass-band [0.1-1] $\mathrm{Hz}$ and spatial filter 


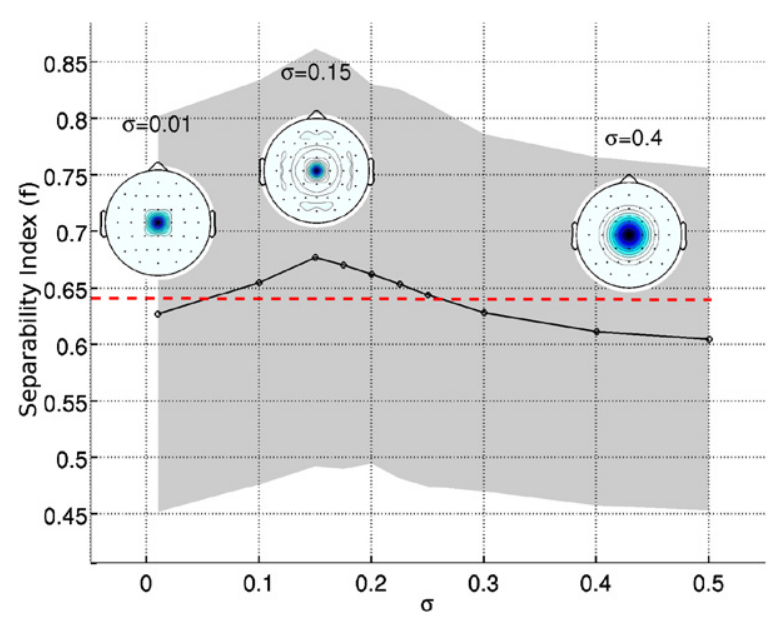

Figure 5. The average $f$ value across 11 subjects for the SSF filter applied at CAR referenced $\mathrm{Cz}$ electrode potentials. The topographies correspond to SSF kernels for $\sigma=0.01,0.15$ and 0.4 . The dotted line represents $f$ values obtained for a spatial filter combination of CAR and WAVG. On average the peak $f$ value was observed at $\sigma=0.15$

CAR+SSF $(\sigma=0.15)$ discussed in sections 4.1 and 4.2. Although [0.3-1] Hz seems to be a better spectral range, we report for the [0.1-1] Hz filter as they resulted in statistically similar $f$ values. To assess the generalization capabilities we trained subject-specific classifiers using the data of day 1 and test on day 2 for all the subjects. Moreover, we also performed these tests using data from day 3 for the two subjects (1 and 3 ) for whom the data were available.

Generalization capabilities. As seen in figure 6, the classifiers showed remarkably high performance when tested on day 2. It is worth noting that even for the lowest performing subject (8), the AUC was $0.81 \pm 0.03$. The best performer (subject 7), achieved $0.97 \pm 0.01$ and the average AUC over the 11 subjects is $0.88 \pm 0.05$. Two out of 11 subjects ( 7 and 10) showed AUC above $0.95,2$ subjects ( 3 and 6 ) fall in the range $0.90-0.95$, and the rest are in the range 0.8-0.9.

The generalization capabilities of the classification techniques are further assessed for day 3 on two subjects (see figure 7). The AUC values for the third day are similar to day 2 with a slight improvement of approximately $2 \%$ for both subjects (the third day recordings for these subjects were separated by a gap of 13 and 7 days from the first session, respectively). These results suggest that the obtained models are robust enough for generalization across days. These stable performances are likely due to the stability of the underlying SCPs features.

\section{Multi-electrode classifier fusion}

The classification results reported in the previous section are based on single electrode $(\mathrm{Cz})$ features alone. However, due to imprecise estimation of parameters, a single classifier is likely to be prone to errors. This error can be reduced by fusing the classifiers computed for each electrode. In this work, we choose a Bayesian fusion technique at the level of classifiers [61]. Alternatively, the fusion could be applied at the level of features. However, the later requires a larger amount of data due to the increased feature dimension. The fusion technique based on Bayes' average is implemented as below.

Given a posterior probability $p^{i}\left(\mathcal{C}_{\mathrm{Go}} \mid \mathbf{x}\right)$ of a classifier computed for an electrode $i$, the fused decision function $\mathcal{D}$ for $K$ of such classifiers is calculated by,

$$
\mathcal{D}\left(\mathbf{x} \in \mathcal{C}_{\mathrm{Go}}\right)=\frac{1}{K} \sum_{i=1}^{K} p^{i}\left(\mathcal{C}_{\mathrm{Go}} \mid \mathbf{x}\right)
$$

This technique is based on the assumption that the individual classifier's posterior probability suffered an error with a zero mean Gaussian distribution. If all the $K$ classifiers are non-random and task-relevant, the Bayes-average fusion is expected to result in a better $\mathcal{D}$.

The mean AUC over all subjects for Bayes-average classifier fusion for different electrode configurations is shown in figure 8(a). The electrode configurations are chosen by the order of increased Euclidean distance from $\mathrm{Cz}$ electrode location. The resulting AUC increases as the number of electrodes are increased from one to nine until most of the fronto-central electrodes are included. The AUC obtained by fusing 5 (with $\mathrm{AUC}=0.89 \pm 0.17)$ and $9(\mathrm{AUC}=0.9 \pm 0.14)$ electrode configurations resulted in a statistically significant improvement over a single electrode $(\mathrm{Cz})$ (Wilcoxon, $p=$ $0.01)$. The AUC decreases as the outer electrodes are included in the fusion. Note that the outer electrodes carry no task-relevant information and the corresponding individual classifiers perform close to random level (see inset of figure 8(a)). The inclusion of such electrodes in the fusion formula impairs the Bayes average. We emphasize that this is a simple classifier fusion technique, other techniques that take into account the classifier's confusion matrix are worth exploring in the future [62].

\section{Artifact influence}

Like any EEG signal, SCPs are prone to artifacts resulting from eye, muscular and tongue movements, as well as skin conductance changes $[18,38,51,50,33,34]$. In this work, the number of movement artifacts is reduced by explicit instructions to avoid blinking or to move. However, it is essential to test whether the classification models exploit such artifacts in case they are systematically introduced with respect to the tasks (i.e., classes). To do so, we computed similar LDA classifiers on the three EOG and EMG derivations and compared their performance to the classifiers built with each EEG electrode (trained using day 1 and tested using day 2 recordings). In all cases, the signals are bandpass filtered in [0.1-1] Hz. Figure 8(b) shows the comparison of AUC of classifiers based on the EOG and EMG derivation with respect to the $\mathrm{Cz}$ electrode. The topographic plot in the inset of figure 8(a) shows the average AUC over 11 subjects for each electrode. From the figures, we observe that the AUC of $\mathrm{Cz}$ is significantly higher than EOG and EMG channels (Wilcoxon, $p=0.01$ ). Indeed, the AUCs of EOG and EMG derivations are close to chance level (Wilcoxon, $p=0.01$ ). Furthermore, the AUCs have high values only at central electrodes, where the anticipatory SCP is known to be prominent, and not at the 

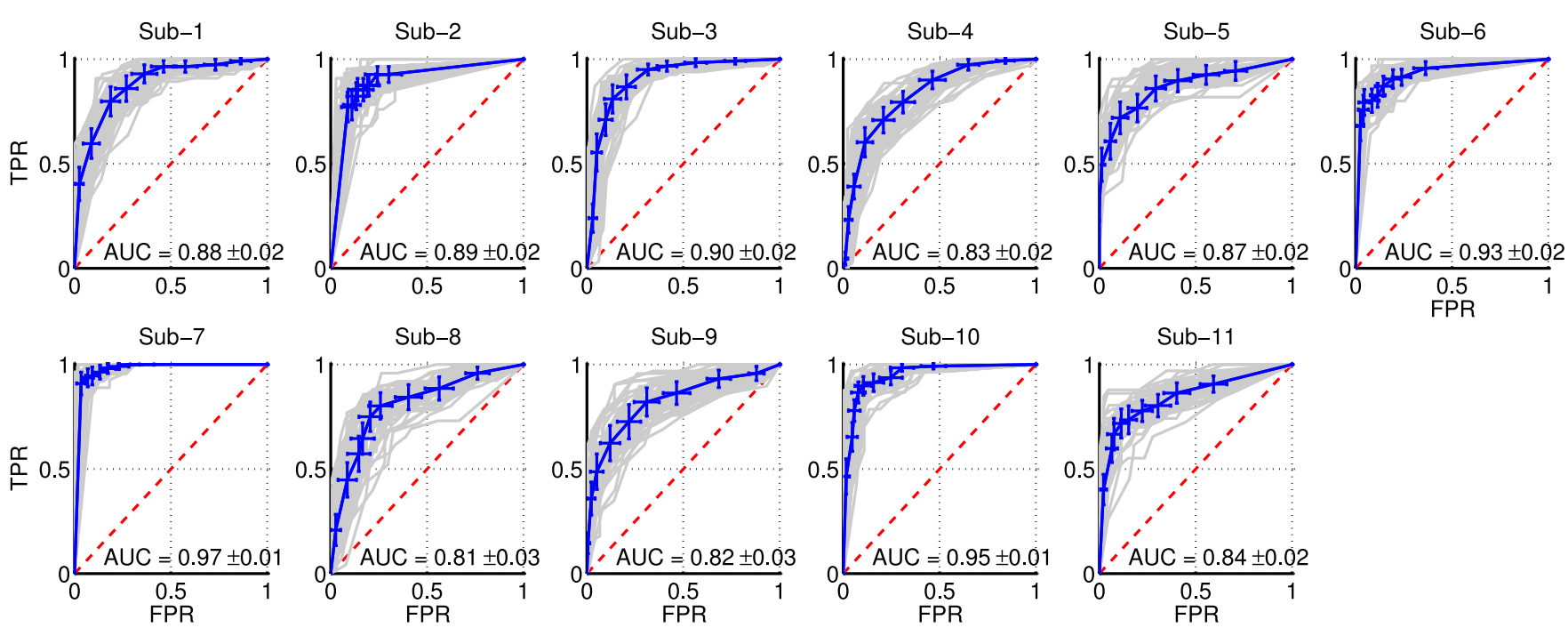

Figure 6. ROC curves and AUC values for the test data of day 2 for 11 subjects (10-fold cross-validation). The red line represents random performance and blue lines represents the ROC curves. The blue "+ " is the standard deviation across folds of FPR along the $x$-axis and that of the TPR along the $y$-axis. The gray lines indicates the corresponding cross validation ROC curves for each of the ten folds. The values of AUCs are shown at the bottom of each ROC curve.
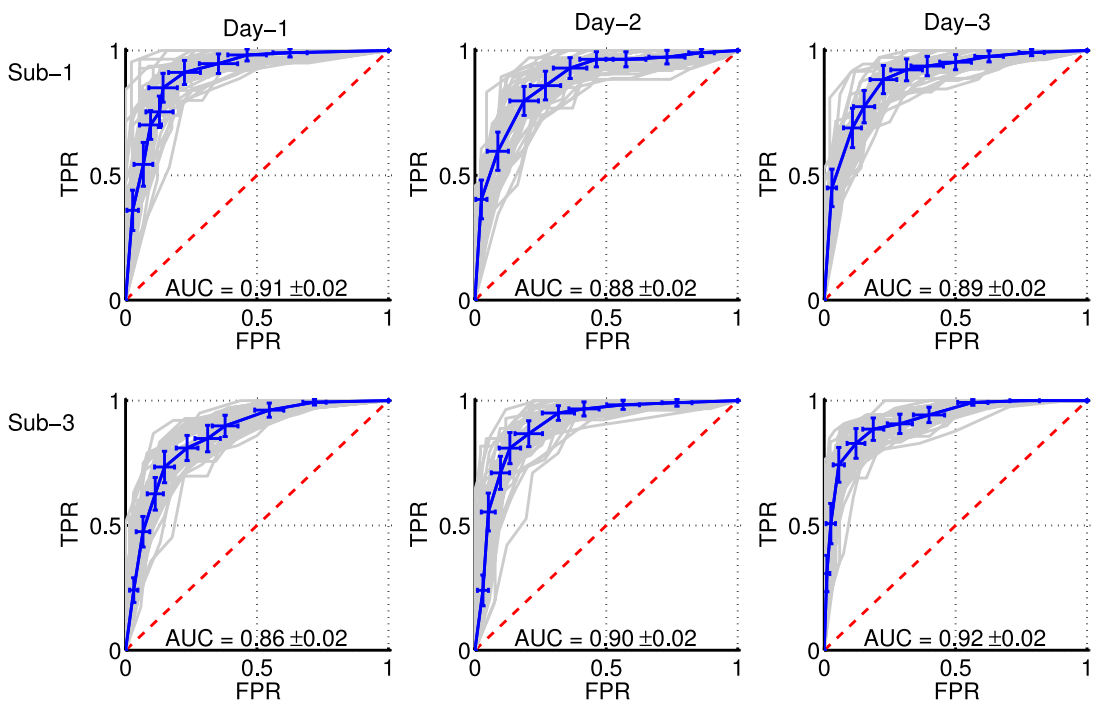

(a)

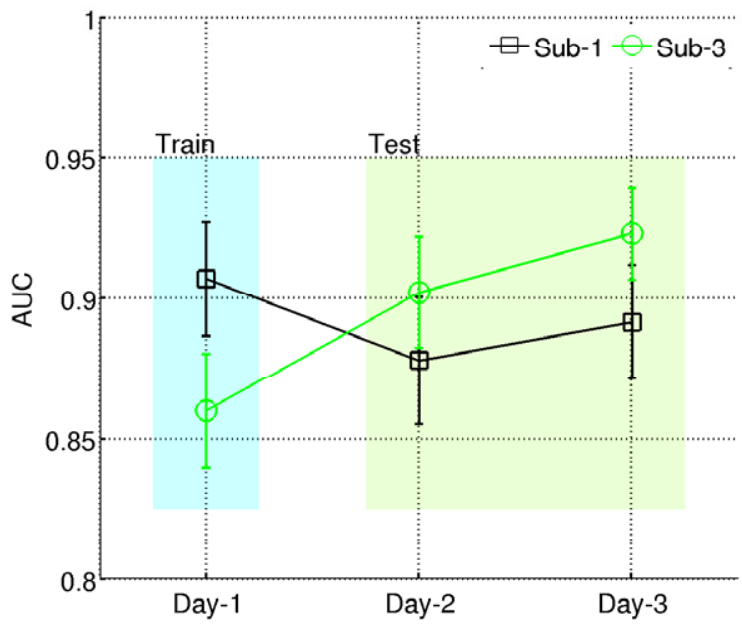

(b)

Figure 7. Generalization capabilities of classifiers for days 2 and 3 for subjects 1 and 3. (a) The ROC curves for day 1 (training data) and for days 2 and 3 (test data). (b) The mean AUC for subjects 1 and 3 for training (day 1) and test data (days 2 and 3) are shown. 


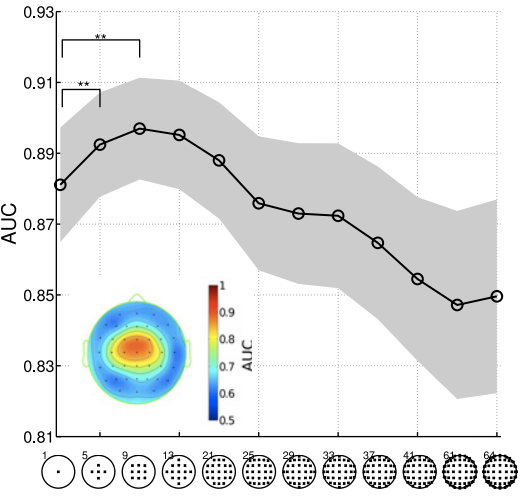

(a)

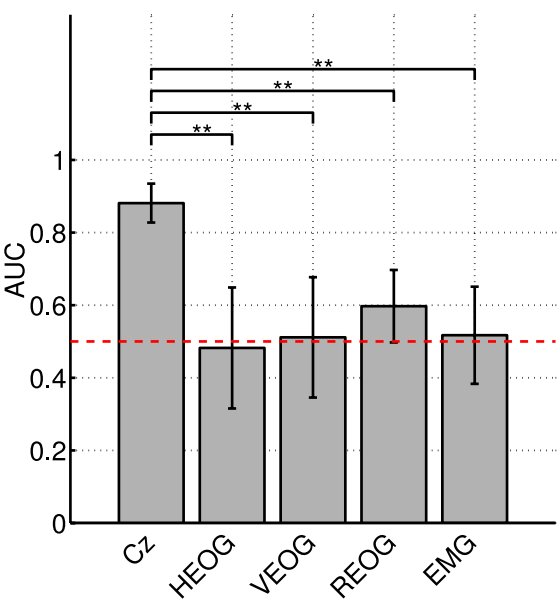

(b)

Figure 8. The mean AUC of 11 of the subjects in various channels and combinations compared. (a) On the $x$-axis, different electrode configurations are shown along with the number of electrodes. The configuration with nine electrodes resulted in the highest AUC being significant as compared to single electrode AUC (Wilcoxon, $p=0.01^{* *}$ ). The AUC of single electrode classifiers is shown topographically in the inset. (b) The mean AUC obtained with $\mathrm{Cz}$ electrode features is compared with horizontal, vertical and radial EOG derivations and EMG features. The red dotted line corresponds to the chance performance level.

outer electrodes (see figures 3). Since the classifiers based on ocular and muscular activity yield no discriminability, it is very unlikely that the EEG classification models exploited such artifacts.

\section{Discussion}

Analysis of SCPs can help us understand various cognitive functions and can be exploited for BCI applications $[2,63,23]$. However, due to the low SNR associated with SCP measurements, most previous studies relied on grandaverages. Thus, there is a need for applying appropriate preprocessing methods that enhance the SNR of SCPs and allow robust single trial analysis (see section 2). In this paper we report the effects of spectral and spatial filter parameters on the analysis of anticipation related SCPs recorded in a realistic AT based web-browsing application. We found that it is more appropriate to bandpass filter the data in the range of [0.1-1] $\mathrm{Hz}$ to [0.3-1] $\mathrm{Hz}$. Such a narrow bandpass filter ${ }^{1}$ yields better separation of the CNV Go/No-go features, as opposed to common practice (i.e., no filtering, just lowpass filtering or minimal high pass filtering with cut-off below $0.05 \mathrm{~Hz}$; see supplementary table 1, available from stacks.iop.org/JNE/10/036014/mmedia). This spectral filter effectively reduces the high amplitude ISO oscillations, and oscillations above the mid-delta band that are task irrelevant (see section 4.1). We also found that widely used spatial filters such as SLAP, LLAP and CAR are sub-optimal compared to CAR in combination with WAVG. The WAVG is implemented as an anti-Laplacian, where neighboring electrode activity is added instead of being subtracted (see section 4.2). We introduced a generalized version of the WAVG filter, SSF, implemented by spatial convolution of the SCP scalp

1 For practical, real-time implementation of the methods, perhaps the best choice is to use the narrow bandpass filter with the range [0.3-1] Hz, which attenuate up to $20 \mathrm{~dB}$ power for frequencies $\leqslant 0.1 \mathrm{~Hz}$. distribution with an isometric Gaussian kernel to effectively reduce the high frequency spatial noise.

The effect of these spatial and spectral filters on the feature distribution is illustrated in figure 9 using the data of subject 1 on day 1 . The figure shows a canonical projection $y$ and features $x_{3}$ and $x_{8}$ described in section 3. When neither spectral nor spatial filters are applied (i.e., raw features) the distributions of Go and No-go have a large overlap and almost no separability can be observed (with $f=0.06$ ). Spectral filtering with a bandpass of [0.1-1] $\mathrm{Hz}$ resulted in a shrinkage of the distribution (note the change in scale), as high amplitude ISO components are reduced and an increased separation of the distributions $(f=0.18)$ is observed. The separability is further enhanced by the application of CAR in combination with SSF for $\sigma=0.15(f=0.74)$.

We demonstrated the generalization capabilities of the spectral $([0.1-1] \mathrm{Hz})$ and the spatial $(\mathrm{CAR}+\mathrm{SSF}$ with $\sigma=$ $0.15)$ filter combination by training subject specific linear classifiers using the recordings of day 1 and testing on the recordings of day 2 (see section 4.3). We reported remarkably high performance with an average AUC over 11 subjects of $0.88 \pm 0.05$ ranging from a minimum of $0.81 \pm 0.03$ for subject 8 to a maximum of $0.97 \pm 0.01$ for subject 7 (see figure 7 (a)). To this end, we assumed a class specific unimodal Gaussian distribution with a shared covariance matrix and stationarity of the features. The high performance of the LDA classifiers suggest that these assumptions are valid to a great extent (see the illustration of the feature distributions for a subject, figure 9).

For subjects 1 and 3, for whom the recordings of day 3 are available, we report stable performances across all sessions, where each session is separated by a gap of 13 and 7 days (see figure 7). These performances suggest the existence of stable SCP features that have been extracted by our filters and captured by the linear classifiers. We also show the possibility of improving the performance with 

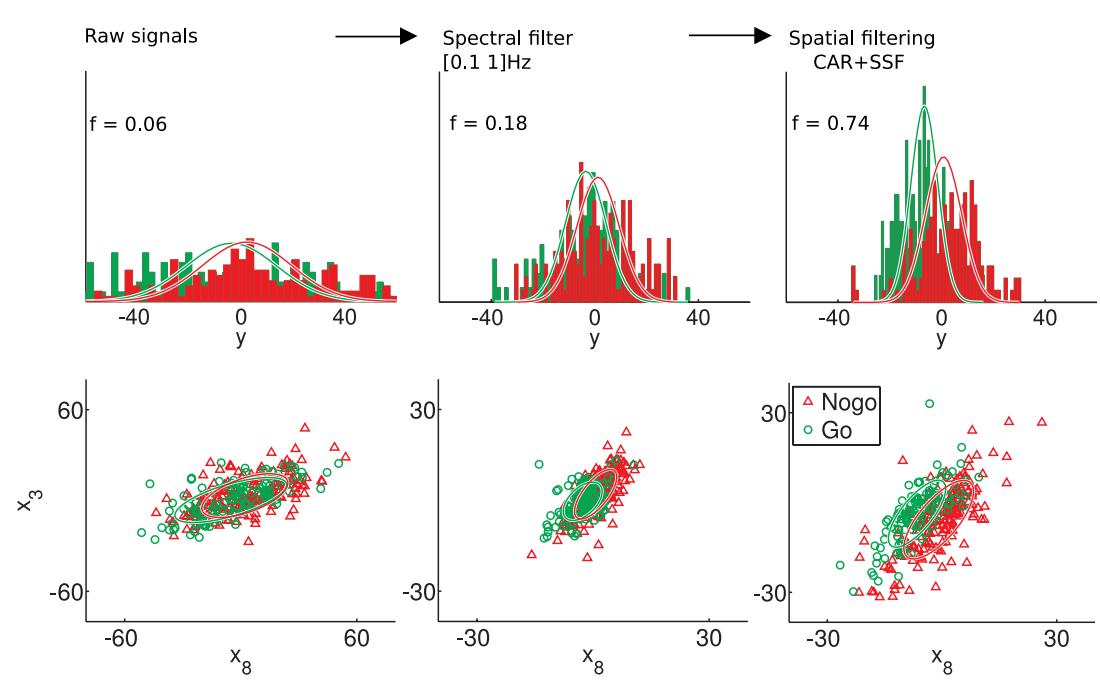

Figure 9. The effect of spectral and spatial filters on feature distributions of CNV Go (green) and No-go (red) features. In the top row the distributions in the canonical space ( $y$; see section 3) are shown for different stages of spectral and spatial filtering. The bottom row shows distribution of features in two dimensions $\left(x_{3}\right.$ and $\left.x_{8}\right)$. The first column corresponds to the features without any spectral filtering referenced to the $\mathrm{Oz}$ electrode. The second column corresponds to features obtained after the application of spectral filters $0.1-1 \mathrm{~Hz}$. The last column shows the feature distributions after the application of both spectral filtering and spatial filtering using CAR+SSF (with $\sigma=0.15$ ). The $f$ values are shown at each stage of preprocessing.

a Bayesian fusion technique that combines multiple single channel classifiers, yielding an increase of approximately $2 \%$. We also controlled that the classification methods were not affected by artifacts such as EOG or EMG.

The SSF introduced for the spatial filtering of SCPs assumed a symmetric distribution around the electrode under study (e.g., the $\mathrm{Cz}$ electrode). In reality this assumption is not fully valid (see figure 3 ). To address the asymmetric nature of SCP distribution, data driven approaches for spatial filtering similar to common spatial patterns (CSP) [64], independent component analysis [65] and beamforming techniques are worth investigating [66]. The performance of SSF with different electrode densities should be compared. In this paper we assume stationarity of the SCP features. However, due to learning effects (suggested by the improved RTs of the experimental subjects with the web browsing protocol, results not reported here), the associated SCP feature distributions could change over time, becoming better separable [23]. Under such conditions, we could resort to adaptation techniques to better model the SCP by tracking the feature distributions [67-69].

\section{Conclusions}

Our study on anticipation related SCPs suggests that it is necessary to bandpass filter the FbEEG around [0.1-1] $\mathrm{Hz}$ to effectively reduce the high amplitude ISOs and non-time locked frequencies above mid-delta oscillations. It is worth noting that the parameters of the spectral filter identified in this study have been successfully applied to other single trial analyses of SCPs such as movement related SCPs [70, 71] and $\mathrm{CNV}$ potentials. Interestingly, this spectral band has also been utilized in decoding movement parameters by other groups [13-15, 72, 45, 46].
To reduce high frequency spatial noise we suggest applying a spatial filter that combines CAR with the spatial smoothing filter. In [73], the method works even if SCPs develops over $1 \mathrm{~s}$. It should be noticed that the requirement of a sharp high-pass cut-off at $0.1 \mathrm{~Hz}$ to reduce ISOs comes with the cost of significant group delay, creating a challenge for applications that require real-time analysis. In this respect, the use of a high-pass cut-off at $0.3 \mathrm{~Hz}$, which yields statistically similar results, could alleviate this problem.

The suggested spatial and spectral methods are aimed at improving the SNR, and thus are not only useful for the single trial analysis but also for the grand-average analysis. Furthermore, we believe these methods are also applicable to any SCPs recorded with EEG and MEG, as well as electrocorticogram (ECoG) analysis. Moreover, our study points out the need for carefully choosing the preprocessing methods when analyzing EEG, a fact often overlooked in the literature (see supplementary table 1, available from stacks.iop.org/JNE/10/036014/mmedia); [74-77]).

\section{Acknowledgments}

The authors are grateful to Dr Robert Leeb, Dr Nicolas Bourdaud, Dr Aleksander Sobolewski, Dr Tom Carlson, Luca Tonin, Michelle Tavella, Serafeim Perdikis and Zahra Khaliliardali for their help during the implementation of the protocol as well as valuable discussions regarding the analysis and proofreading.

\section{References}

[1] Birbaumer N, Elbert T, Canavan A G and Rockstroh B 1990 Slow potentials of the cerebral cortex and behavior Physiol. Rev. 70 1-41 (PMID: 2404287)

[2] Birbaumer N, Hinterberger T, Kübler A and Neumann N 2003 The thought-translation device (TTD): neurobehavioral 
mechanisms and clinical outcome IEEE Trans. Neural Syst. Rehabil. Eng. 11 120-3

[3] Altenmülter E O, Münte T F and Gerloff C 2011 Neurocognitive functions and the EEG Niedermeyer's Electroencephalography: Basic Principles, Clinical Applications and Related Fields 6th edn, ed D L Schomer and F H Lopes da Silva (Philadelphia: Wolters Kluwer/ Lippincott Williams \& Wilkins) pp 661-82

[4] Marjan J and Mark H 2003 The Bereitschaftspotential: Movement-Related Cortical Potentials (New York: Kluwer)

[5] Walter W G, Cooper R, Aldridge V J, McCallum W C and Winter A L 1964 Contingent negative variation: an electric sign of sensorimotor association and expectancy in the human brain Nature 203 380-4

[6] Green A M and Kalaska J F 2011 Learning to move machines with the mind Trends Neurosci. 34 61-75

[7] Millán J d R et al 2010 Combining brain-computer interfaces and assistive technologies: state-of-the-art and challenges Front. Neurosci. 4161

[8] Wolpaw J R, Birbaumer N, McFarland D J, Pfurtscheller G and Vaughan T M 2002 Brain-computer interfaces for communication and control Clin. Neurophysiol. $113767-91$

[9] Waldemar K and Erwin H 2010 ERP correlates of linear hand movements: distance dependent changes Clin. Neurophysiol. 121 1285-92

[10] Waldemar K, Erwin H and Frank R 2010 ERP correlates of linear hand movements in a motor reproduction task Psychophysiology 47 486-500

[11] Ying G, Kim D and Dario F 2009 Single-trial discrimination of type and speed of wrist movements from EEG recordings Clin. Neurophysiol. 120 1596-600

[12] Ying G, Dario F, Ramos M A, Kim D, Pedro M and Birbaumer N 2009 Offline identification of imagined speed of wrist movements in paralyzed ALS patients from single-trial EEG Front. Neurosci. 362

[13] Nascimento d O F and Farina D 2008 Movement-related cortical potentials allow discrimination of rate of torque development in imaginary isometric plantar flexion IEEE Trans. Biomed. Eng. 55 2675-8

[14] Bradberry T J, Gentili R J and Contreras-Vidal J L 2010 Reconstructing three-dimensional hand movements from noninvasive electroencephalographic signals J. Neurosci. 30 3432-7

[15] Agashe H A and Contreras-Vidal J L 2011 Reconstructing hand kinematics during reach to grasp movements from electroencephalographic signals Conf. Proc. IEEE Eng. Med. Biol. Soc. 2011 5444-7

[16] Lu M-K, Arai N, Tsai C-H and Ziemann U 2012 Movement related cortical potentials of cued versus self-initiated movements: double dissociated modulation by dorsal premotor cortex versus supplementary motor area rTMS Hum. Brain Mapp. 33 824-39

[17] Platz T, Kim I H, Pintschovius H, Winter T, Kieselbach A, Villringer K, Kurth R and Mauritz K H 2000 Multimodal EEG analysis in man suggests impairment-specific changes in movement-related electric brain activity after stroke Brain 123 2475-90

[18] Lutzenberger W, Elbert T, Rockstroh B and Birbaumer N 1980 Biofeedback of slow cortical potentials. II. Analysis of single event-related slow potentials by time series analysis Electroencephalogr. Clin. Neurophysiol. 48 302-11

[19] Kübler A, Kotchoubey B, Hinterberger T, Ghanayim N, Perelmouter J, Schauer M, Fritsch C, Taub E and Birbaumer N 1999 The thought translation device: a neurophysiological approach to communication in total motor paralysis Exp. Brain Res. 124 223-32

[20] Birbaumer N, Ghanayim N, Hinterberger T, Iversen I, Kotchoubey B, Kübler A, Perelmouter J, Taub E and Flor H
1999 A spelling device for the paralysed Nature 398 297-8

[21] Schneider F, Heimann H, Mattes R, Lutzenberger W and Birbaumer N 1992 Self-regulation of slow cortical potentials in psychiatric patients: depression Biofeedback Self-Regul. 17 203-14

[22] Garipelli G, Chavarriaga R and Millán J d R 2009 Fast recognition of anticipation-related potentials IEEE Trans. Biomed. Eng. 56 1257-60

[23] Garipelli G, Chavarriaga R and Millán J 2009 Anticipation based brain-computer interfacing (aBCI) Proc. 4th Int. IEEE EMBS Conf. on Neural Engineering pp 459-62

[24] Garipelli G, Chavarriaga R, Cincotti F, Babiloni F and Millán J d R 2009 Discriminative channel selection method for the recognition of anticipation related potentials from CCD estimated cortical activity IEEE Int. Machine Learning for Signal Processing Workshop pp 1-6

[25] Blankertz B, Lemm S, Treder N, Haufe S and Müller K-R 2011 Single-trial analysis and classification of ERP components-a tutorial Neuroimage 56 814-25

[26] Parra L C, Spence C D, Gerson A D and Sajda P 2005 Recipes for the linear analysis of EEG Neuroimage 28 326-41

[27] Bashashati A, Fatourechi M, Ward R K and Birch G E 2007 A survey of signal processing algorithms in brain-computer interfaces based on electrical brain signals J. Neural Eng. $432-57$

[28] Bai O, Rathi V, Lin P, Huang D, Battapady H, Fei D-Y, Schneider L, Houdayer E, Chen X and Hallett M 2011 Prediction of human voluntary movement before it occurs Clin. Neurophysiol. 122 364-72

[29] Sanchez-Vives M V and McCormick D A 2000 Cellular and network mechanisms of rhythmic recurrent activity in neocortex Nature Neurosci. 3 1027-34

[30] Steriade M 1993 Central core modulation of spontaneous oscillations and sensory transmission in thalamocortical systems Curr. Opin. Neurobiol. 3 619-25

[31] Timofeev I, Grenier F, Bazhenov M, Sejnowski T J and Steriade M 2000 Origin of slow cortical oscillations in deafferented cortical slabs Cereb. Cortex 10 1185-99

[32] Bauer H, Korunka C and Leodolter M 1989 Technical requirements for high-quality scalp DC recordings Electroencephalogr. Clin. Neurophysiol. 72 545-7

[33] Vanhatalo S, Voipio J and Kaila K 2005 Full-band EEG (FbEEG): an emerging standard in electroencephalography Clin. Neurophysiol. 116 1-8

[34] Voipio J, Tallgren P, Heinonen E, Vanhatalo S and Kaila K 2003 Millivolt-scale DC shifts in the human scalp EEG: evidence for a nonneuronal generator J. Neurophysiol. 89 2208-14

[35] Buzsáki G 2006 Rhythms of the Brain 1st edn (Oxford: Oxford University Press)

[36] Demanuele C, James C J and Sonuga-Barke E J S 2007 Distinguishing low frequency oscillations within the $1 / \mathrm{f}$ spectral behaviour of electromagnetic brain signals Behav. Brain Funct. 362

[37] Penttonen M and Buzsáki G 2003 Natural logarithmic relationship between brain oscillators Thalamus Relat. Syst. 2 145-52

[38] Picton T W and Hillyard S A 1972 Cephalic skin potentials in electroencephalography Electroencephalogr. Clin. Neurophysiol. 33 419-24

[39] Babiloni C, Brancucci A, Capotosto P, Romani G L, Arendt-Nielsen L, N Chen A C and Rossini P M 2005 Slow cortical potential shifts preceding sensorimotor interactions Brain Res. Bull. 65 309-16

[40] Christopher A B, Ben S, Yvonne B W E-D and Anthony K P J 2008 Modulation of pain ratings by expectation and uncertainty: behavioral characteristics and anticipatory neural correlates Pain 135 240-50 
[41] Filipović S R, Jahanshahi M and Rothwell J C 2001 Uncoupling of contingent negative variation and alpha band event-related desynchronization in a go/no-go task Clin. Neurophysiol. 112 1307-15

[42] Morash V, Bai O, Furlani S, Lin P and Hallett M 2008 Classifying EEG signals preceding right hand, left hand, tongue, and right foot movements and motor imageries Clin. Neurophysiol. 119 2570-8

[43] Travis F and Tecce J J 1998 Effects of distracting stimuli on CNV amplitude and reaction time Int. J. Psychophysiol. 31 45-50

[44] Hamano T, Lüders H O, Ikeda A, Collura T F, Comair Y G and Shibasaki H 1997 The cortical generators of the contingent negative variation in humans: a study with subdural electrodes Electroencephalogr. Clin. Neurophysiol. 104 257-68

[45] Presacco A, Goodman R, Forrester L and Contreras-Vidal J L 2011 Neural decoding of treadmill walking from noninvasive electroencephalographic signals J. Neurophysiol. 106 1875-87

[46] Niazi I K, Jiang N, Tiberghien O, Nielsen J Feldbk, Dremstrup K and Farina D 2011 Detection of movement intention from single-trial movement-related cortical potentials J. Neural Eng. 8066009

[47] Hinterberger T, Schmidt S, Neumann N, Mellinger J, Blankertz B, Curio G and Birbaumer N 2004 Brain-computer communication and slow cortical potentials IEEE Trans. Biomed. Eng. 51 1011-8

[48] Klein C, Andresen B, Berg P, Krüger H and Rockstroh B 1998 Topography of CNV and PINV in schizotypal personality Psychophysiology 35 272-82

[49] MacKay W A and Bonnet M 1990 CNV, stretch reflex and reaction time correlates of preparation for movement direction and force Electroencephalogr. Clin. Neurophysiol. 76 47-62

[50] Vanhatalo S, Tallgren P, Becker C, Holmes M D, Miller J W, Kaila K and Voipio J 2003 Scalp-recorded slow EEG responses generated in response to hemodynamic changes in the human brain Clin. Neurophysiol. 114 1744-54

[51] Vanhatalo S, Voipio J, Dewaraja A, Holmes M D and Miller J W 2003 Topography and elimination of slow EEG responses related to tongue movements Neuroimage 20 1419-23

[52] Rockstroh B, Elbert T, Canavan A, Lutzenberger W and Birbaumer N 1989 Slow Cortical Potentials and Behaviour 2nd edn (Baltimore: Urban and Schwarzenberg)

[53] Friedrich E V C, McFarland D J, Neuper C, Vaughan T M, Brunner P and Wolpaw J R 2009 A scanning protocol for sensorimotor rhythm-based brain-computer interface Biol. Psychol. 80 169-75

[54] Croft R J and Barry R J 2000 Removal of ocular artifact from the EEG: a review Neurophysiol. Clin. $305-19$

[55] Schlögl A, Keinrath C, Zimmermann D, Scherer R, Leeb R and Pfurtscheller G 2007 A fully automated correction method of EOG artifacts in EEG recordings Clin. Neurophysiol. 118 98-104

[56] McFarland D J, McCane L M, David S V and Wolpaw J R 1997 Spatial filter selection for EEG-based communication Electroencephalogr. Clin. Neurophysiol. 103 386-94

[57] Lindquist M A and Wager T D 2008 Spatial smoothing in fMRI using prolate spheroidal wave functions Hum. Brain Mapp. 29 1276-87

[58] Duda R O, Hart P E and Stork D G 2000 Pattern Classification 2nd edn (New York: Wiley-Interscience)
[59] Fawcett T 2006 An introduction to ROC analysis Pattern Recognit. Lett. 27 861-74

[60] Hanley J A and McNeil B J 1982 The meaning and use of area under a receiver operating characteristic (ROC) curve Radiology 143 29-36 (PMID: 7063747)

[61] Tax D M J, van Breukelen M, Duin R P W and Kittler J 2000 Combining multiple classifiers by averaging or by multiplying? Pattern Recognit. 33 1475-85

[62] Kittler J, Hatef M, Duin R P W and Matas J 1998 On combining classifiers IEEE Trans. Pattern Anal. Mach. Intell. 20 226-39

[63] Kübler A, Neumann N, Kaiser J, Kotchoubey B, Hinterberger T and Birbaumer N P 2001 Brain-computer communication: self-regulation of slow cortical potentials for verbal communication Arch. Phys. Med. Rehabil. 82 1533-9

[64] Lemm S, Blankertz B, Curio G and Müller K-R 2005 Spatio-spectral filter for improved classification of single-trial EEG IEEE Trans. Biomed. Eng. 52 1541-8

[65] Hyvärinen A and Oja E 2000 Independent component analysis: algorithms and applications Neural Netw. 13 411-30

[66] Grosse-Wentrup M, Liefhold C, Gramann K and Buss M 2009 Beamforming in noninvasive brain-computer interfaces IEEE Trans. Biomed. Eng. 56 1209-19

[67] Buttfield A, Ferrez W P and Millán J d R 2006 Towards a robust BCI: error potentials and online learning IEEE Trans. Neural Syst. Rehabil. Eng. 14 164-8

[68] Millán J d R et al 2007 Adaptation in brain-computer interfaces Toward Brain-Computer Interfacing ed G Dornhege, Jd R Millán, T Hinterberger, D McFarland and K R Müller (Cambridge, MA: MIT press) pp 303-25

[69] Vidaurre C, Kawanabe M, von Bünau P, Blankertz B and Müller K-R 2011 Toward unsupervised adaptation of LDA for brain-computer interfaces IEEE Trans. Biomed. Eng. 58 587-97

[70] Lew Y L E, Chavarriaga R, Silvoni S and Millán J d R 2012 Detection of self-paced reaching movement intention from EEG signals Front. Neuroeng. 513

[71] Lew Y L E, Chavarriaga R, Zhang H, Seeck M and Millán J d R 2012 Self-paced movement intention detection from human brain signals: invasive and non-invasive EEG 34th Ann. Int. Conf. of the IEEE Engineering in Medicine and Biology (San Diego, USA) pp 3280-3

[72] Bradberry T J, Gentili R J and Contreras-Vidal J L 2009 Decoding three-dimensional hand kinematics from electroencephalographic signals Proc. Int. Conf. of the IEEE Engineering in Medicine and Biology Society vol 2009, pp 5010-3

[73] Khaliliardali Z, Chavarriaga R, Gheorghe L A and Millán J d R 2012 Detection of anticipatory brain potentials during car driving Proc. 34th Annu. Int. Conf. of the IEEE Engineering in Medicine and Biology Society pp 3829-32

[74] Acunzo D J, Mackenzie G and van Rossum M C W 2012 Systematic biases in early ERP and ERF components as a result of high-pass filtering J. Neurosci. Methods 209 212-8

[75] Vanrullen R 2011 Four common conceptual fallacies in mapping the time course of recognition Front. Psychol. 2365

[76] Rousselet G A 2012 Does filtering preclude Us from studying ERP time-courses? Front. Psychol. 3131

[77] Widmann A and Schröger E 2012 Filter effects and filter artifacts in the analysis of electrophysiological data Front. Psychol. 3233 